\title{
Single-photon nonlinearities in two-mode optomechanics
}

\author{
P. Kómár, ${ }^{1}$ S. D. Bennett, ${ }^{1}$ K. Stannigel, ${ }^{2}$ S. J. M. Habraken, ${ }^{2,3}$ P. Rabl, ${ }^{4}$ P. Zoller,,${ }^{2,3}$ and M. D. Lukin ${ }^{1}$ \\ ${ }^{1}$ Physics Department, Harvard University, Cambridge, Massachusetts 02138, USA \\ ${ }^{2}$ Institute for Quantum Optics and Quantum Information, Austrian Academy of Sciences, 6020 Innsbruck, Austria \\ ${ }^{3}$ Institute for Theoretical Physics, University of Innsbruck, 6020 Innsbruck, Austria \\ ${ }^{4}$ Institute of Atomic and Subatomic Physics, TU Wien, Stadionallee 2, 1020 Wien, Austria
}

(Received 17 October 2012; published 28 January 2013)

\begin{abstract}
We present a detailed theoretical analysis of a weakly driven, multimode optomechanical system, in which two optical modes are strongly and near-resonantly coupled to a single mechanical mode via a three-wave mixing interaction. We calculate one- and two-time intensity correlations of the two optical fields and compare them to analogous correlations in atom-cavity systems. Nonclassical photon correlations arise when the optomechanical coupling $g$ exceeds the cavity decay rate $\kappa$, and we discuss signatures of one- and two-photon resonances as well as quantum interference. We also find a long-lived correlation that decays slowly with the mechanical decay rate $\gamma$, reflecting the heralded preparation of a single-phonon state after detection of a photon. Our results provide insight into the quantum regime of multimode optomechanics, with potential applications for quantum information processing with photons and phonons.
\end{abstract}

DOI: 10.1103/PhysRevA.87.013839

PACS number(s): 42.50.Pq, 42.50.Ct, 42.50.Wk, 07.10.Cm

\section{INTRODUCTION}

Optomechanical systems (OMSs) involve the interaction between optical and mechanical modes arising from radiation pressure force, canonically in an optical cavity with a movable mirror [1-5]. Recent progress in optomechanical (OM) cooling techniques has been rapid [6-13], and experiments have now demonstrated cooling to the mechanical ground state [14-16], OM-induced transparency [17,18], and coherent photon-phonon conversion [19-21]. These developments have attracted significant interest, and motivated proposals for applications exploiting OM interactions at the quantum level, ranging from quantum transducers [22-25] and mechanical storage of light [26-28] to single-photon sources [29] and OM quantum information processing [30,31]. Significant advantages of OM platforms for these applications are the possibility for mass production and on-chip integration using nanofabrication technologies, wide tuneability, and the versatility of mechanical oscillators to couple to a wide variety of quantum systems [32].

The force exerted by a single photon on a macroscopic object is typically weak; consequently, experiments have so far focused on the regime of strong optical driving, where the OM interaction is strongly enhanced but effectively linear [33,34]. However, recent progress in the design of nanoscale OMSs [16,35-37] and OM experiments in cold-atomic systems $[38,39]$ suggests that the regime of single-photon strong coupling, where the OM coupling strength $g$ exceeds the optical cavity decay rate $\kappa$, is within reach of the next generation of $\mathrm{OM}$ experiments. In this regime, the inherently nonlinear OM interaction is significant at the level of single photons and phonons [29,40-42]. For example, the presence of a single photon can-via the mechanical mode-strongly influence or even block the transmission of a second photon, leading to photon blockade. This single-photon nonlinearity was recently analyzed for canonical OMSs consisting of a single optical mode coupled to a mechanical mode $[29,43,44]$. However, with a single optical mode, the OM coupling is highly off-resonant, leading to a suppression of effective photon-photon interactions by the large mechanical frequency $\omega_{m} \gg g$ [29].

In this paper, we develop a quantum theory of a weakly driven two-mode OMS [30,45-47] in which two optical modes are coupled to a mechanical mode. The key advantage of this approach is that photons in the two optical modes can be resonantly exchanged by absorbing or emitting a phonon via three-mode mixing. We extend our earlier results [30], where we discussed possible applications of resonant optomechanics such as single-photon sources and quantum gates by exploring one-time and two-time photon correlations of both optical modes. Specifically, we find that the photon-photon correlation function of the undriven optical mode exhibits delayed bunching for long delay times, arising from a heralded single mechanical excitation after detection of a photon in the undriven mode. Finally, we compare the two-mode OMS to the canonical atomic cavity QED system with a similar lowenergy level spectrum $[48,49]$. Despite several similarities, we find that in stark contrast to the atom-cavity system, the OMS studied here does not exhibit nonclassical correlations unless the strict strong-coupling condition $g>\kappa$ is met. Our results serve as a guideline for $\mathrm{OM}$ experiments nearing the regime of single-photon nonlinearity, and for potential quantum information processing applications with photons and phonons.

The remainder of the paper is organized as follows. In Sec. II, we introduce the system and details of the model. In Sec. III, we calculate the equal-time intensity correlation functions of both transmitted and reflected photons, and discuss signatures of nonclassical photon statistics. In Sec. IV, we investigate two-time correlation functions of the transmitted photons, and discuss delayed coincidence correlations that indicate the heralded preparation of a single-phonon state. Finally, we provide a brief outlook on the feasibility of strong $\mathrm{OM}$ coupling in Sec. V, and conclude in Sec. VI with a summary of our results. Appendix B contains details of our analytic model used to derive several results discussed in the paper. 
(a)

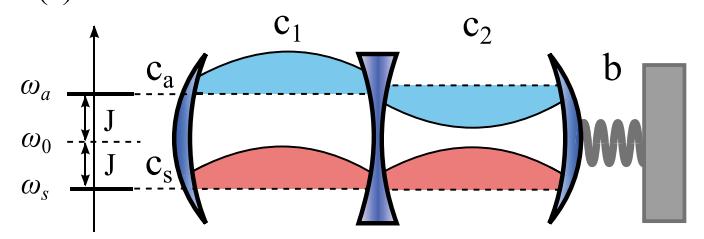

(b)

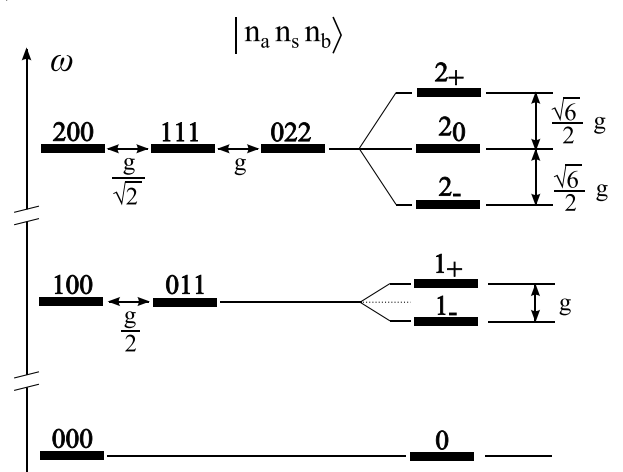

FIG. 1. (Color online) (a) Optomechanical system consisting of two tunnel-coupled optical cavity modes $c_{1}$ and $c_{2}$, and a mechanical oscillator $b$ coupled to one of the cavity modes by radiation pressure. The coupled optical modes are diagonalized in terms of symmetric and antisymmetric modes, $c_{s}$ and $c_{a}$. (b) Level diagram showing the relevant zero-, one- and two-photon states at zero temperature and under the three-mode resonance condition $\omega_{s}-\omega_{a}=\omega_{m}$. States are labeled by $\left|n_{a} n_{s} n_{b}\right\rangle$ denoting the number $n_{a}\left(n_{s}\right)$ of antisymmetric (symmetric) photons and the number of phonons $n_{b}$. The optomechanical coupling $g$ splits the degeneracy of states $\left|n_{a} n_{s} n_{b}\right\rangle$ and $\left|n_{a}-1, n_{s}+1, n_{b}+1\right\rangle$.

\section{MULTIMODE OPTOMECHANICS}

We consider the setup shown schematically in Fig. 1(a), consisting of two optical cavities separated by a semitransparent mirror. The cavity modes are coupled by photons tunneling through the fixed mirror in the middle, and the mode on the right couples to the motion of a vibrating end mirror through radiation pressure. The Hamiltonian describing the system is $(\hbar=1)$

$$
\begin{aligned}
H_{0}= & \omega_{0}\left(c_{1}^{\dagger} c_{1}+c_{2}^{\dagger} c_{2}\right)-J\left(c_{1}^{\dagger} c_{2}+c_{1}^{\dagger} c_{2}\right)+\omega_{m} b^{\dagger} b \\
& -g\left(b^{\dagger}+b\right) c_{2}^{\dagger} c_{2}+H_{\mathrm{dr}}(t),
\end{aligned}
$$

where $c_{1,2}$ are annihilation operators for the two cavity modes, which we assume to be degenerate with frequency $\omega_{0}$, and $J$ is the photon tunneling amplitude through the central mirror. The motion of the end mirror on the right is described by a single mechanical mode with annihilation operator $b$ and frequency $\omega_{m}$, and the parametric coupling strength $g$ corresponds to the shift of the cavity frequency due to a single mechanical phonon. Finally, $H_{\mathrm{dr}}(t)=\sum_{i=1,2}\left(\Omega_{i} c_{i} e^{i \omega_{L} t}+\right.$ H.c. $)$ describes two coherent driving fields of amplitudes $\Omega_{i}$ and frequency $\omega_{L}$, which are applied to the left and right cavities.

We are interested in a three-mode resonant interaction in which the two optical modes exchange a photon by absorbing or emitting a phonon in the mechanical mode. We begin by diagonalizing the optical part of $H_{0}$ in the first line of Eq. (1) in terms of the symmetric and antisymmetric combinations of the optical modes, $c_{s}=\frac{1}{\sqrt{2}}\left(c_{1}+c_{2}\right)$ and $c_{a}=\frac{1}{\sqrt{2}}\left(c_{1}-c_{2}\right)$, with eigenfrequencies $\omega_{a, s}=\omega_{0} \pm J$. In the frame rotating at the laser frequency $\omega_{L}$, we obtain

$$
\begin{aligned}
H^{\prime}= & -\Delta c_{a}^{\dagger} c_{a}-(\Delta+2 J) c_{s}^{\dagger} c_{s}+\omega_{m} b^{\dagger} b \\
& +\frac{g}{2}\left(c_{a}^{\dagger} c_{s}+c_{s}^{\dagger} c_{a}\right)\left(b+b^{\dagger}\right)+\sum_{\eta=s, a} \Omega_{\eta}\left(c_{\eta}^{\dagger}+c_{\eta}\right),
\end{aligned}
$$

where $\Delta=\omega_{L}-\omega_{a}$ is the laser detuning from the $c_{a}$ mode, and $\Omega_{s, a}=\left(\Omega_{1} \pm \Omega_{2}\right) / \sqrt{2}$. Next, we focus on the case of three-mode resonance, $\omega_{m}=2 J$, and assume $\omega_{m} \gg$ $g,|\Delta|, \Omega_{i}$. The third inequality here corresponds to a weak optical drive, which is the relevant limit for studying singlephoton nonlinear effects in this work. This allows us to make a rotating-wave approximation with respect to the remaining large frequency scale $\omega_{m}$, and in the frame defined by the transformation $U=\exp \left[-i \omega_{m} t\left(b^{\dagger} b-c_{s}^{\dagger} c_{s}\right)\right]$, the Hamiltonian $H^{\prime}$ simplifies to

$$
H=-\Delta\left(c_{a}^{\dagger} c_{a}+c_{s}^{\dagger} c_{s}\right)+\frac{g}{2}\left(c_{a}^{\dagger} c_{s} b+b^{\dagger} c_{s}^{\dagger} c_{a}\right)+\Omega_{a}\left(c_{a}^{\dagger}+c_{a}\right) .
$$

This is the starting point for our analysis discussed below. Note that the assumptions made in deriving Eq. (3) are fulfilled in most experimental systems of interest [50,51], and $H$ represents a generic description for resonant two-mode optomechanics [45,46,52,53].

The nonlinear terms proportional to $g$ in Eq. (3) describe coherent photon exchange between the two optical modes mediated by absorption or emission of a phonon. The resulting low-energy level diagram is shown in Fig. 1(b), where $\left|n_{a} n_{s} n_{b}\right\rangle$ represents a state with $n_{a}$ and $n_{s}$ photons in the $c_{a}$ and $c_{s}$ modes, and $n_{b}$ phonons in the mechanical mode. In the absence of a drive, we diagonalize $H$ in this few-photon subspace, yielding the eigenstates

$$
\begin{gathered}
|0\rangle=|000\rangle, \\
\left|1_{ \pm}\right\rangle=\frac{1}{\sqrt{2}}(|100\rangle \pm|011\rangle), \\
\left|2_{ \pm}\right\rangle=\frac{1}{\sqrt{6}}(|200\rangle \pm \sqrt{3}|111\rangle+\sqrt{2}|022\rangle), \\
\left|2_{0}\right\rangle=\frac{1}{\sqrt{3}}(\sqrt{2}|200\rangle-|022\rangle) .
\end{gathered}
$$

Note that in the diagonal basis, the weak driving field couples all states with photon number differing by one. In the following sections, we use this low-energy Hilbert space to understand photon correlations in the system.

In addition to the coherent evolution modeled by the Hamiltonian $H$, we describe optical and mechanical dissipation using a master equation for the system density operator $\rho$,

$$
\begin{aligned}
\dot{\rho}= & -i[H, \rho]+\kappa \mathcal{D}\left[c_{a}\right] \rho+\kappa \mathcal{D}\left[c_{s}\right] \rho \\
& +\frac{\gamma}{2}\left(N_{\mathrm{th}}+1\right) \mathcal{D}[b] \rho+\frac{\gamma}{2} N_{\mathrm{th}} \mathcal{D}\left[b^{\dagger}\right] \rho,
\end{aligned}
$$

where $H$ is given by Eq. (3), $2 \kappa$ and $\gamma$ are energy decay rates for the optical and mechanical modes, respectively, 
$N_{\text {th }}$ is the thermal phonon population, and $\mathcal{D}[\hat{o}] \rho=2 \hat{o} \rho \hat{o}^{\dagger}-$ $\hat{o}^{\dagger} \hat{o} \rho-\rho \hat{o}^{\dagger} o$. Below we study nonlinear effects at the level of single photons, both numerically and analytically, by solving Eq. (8) approximately in the limit of weak optical driving, $\Omega \equiv \Omega_{a} \ll \kappa$.

\section{EQUAL-TIME CORRELATIONS}

\section{A. Average transmission and reflection}

Before focusing on photon-photon correlations, we first study the average transmission through the cavity, which is proportional to the mean intracavity photon number. In Figs. 2(a) and 2(b), we show the intracavity photon number of the two optical modes,

$$
\bar{n}_{i}=\left\langle c_{i}^{\dagger} c_{i}\right\rangle,
$$

where $i=a, s$, and angle brackets denote the steady-state average. At $\Delta / g= \pm \frac{1}{2}$, both transmission curves exhibit a maximum, indicating that the driving field is in resonance with an eigenmode of the system. The position of these peaks can be understood from the level diagram shown in Fig. 1(b), which at finite $g$ shows a splitting of the lowest photonic states into a doublet, $\left|1_{ \pm}\right\rangle=(|100\rangle \pm|011\rangle) / \sqrt{2}$.

In addition to the transmission, we plot the mean reflected photon number in Fig. 2(c). As discussed below, the reflected photon statistics can also exhibit signatures of nonlinearity. We calculate properties of the reflected light using the annihilation operator $c_{R}=c_{a}+i \frac{\Omega}{\kappa}$, obtained from standard input-output relations for a symmetric two-sided cavity (see Appendix A). The mean reflected photon number $\bar{n}_{R}=\left\langle c_{R}^{\dagger} c_{R}\right\rangle$ is plotted in Fig. 2(c). At $\Delta / g= \pm \frac{1}{2}$, the average reflection has a minimum where the average transmission has a maximum. Note that in contrast to a single cavity, even on resonance the transmission probability is less than unity and the reflection probability remains finite.

\section{B. Intensity correlations}

To characterize nonclassical photon statistics in the light transmitted through the OMS, we study the equal-time photonphoton correlation functions,

$$
g_{i i}^{(2)}(0)=\frac{\left\langle c_{i}^{\dagger} c_{i}^{\dagger} c_{i} c_{i}\right\rangle}{\left\langle c_{i}^{\dagger} c_{i}\right\rangle^{2}},
$$

where all operators are evaluated at the same time and $i=a, s, R$. A normalized correlation of $g_{i i}^{(2)}(0)<1$ indicates photon antibunching, and the limit $g_{i i}^{(2)}(0) \rightarrow 0$ corresponds to the complete photon blockade regime in which two photons never occupy the cavity at the same time. The solid curves in Fig. 2 show $g_{a a}^{(2)}(0), g_{s s}^{(2)}(0)$, and $g_{R R}^{(2)}(0)$ as a function of the laser detuning and in the limit of weak driving, $\Omega / \kappa \ll 1$. The most pronounced features of these correlation functions occur at $|\Delta| / g=0, \frac{1}{\sqrt{8}}, \frac{1}{2}$, and $\frac{\sqrt{6}}{4}$, as marked by dots A, B, C, and D, respectively. As we explain in detail in the following analysis, we find that the photon bunching at A and antibunching at B are the result of destructive quantum interference, while the features at points $\mathrm{C}$ and $\mathrm{D}$ arise from one- and two-photon resonances.
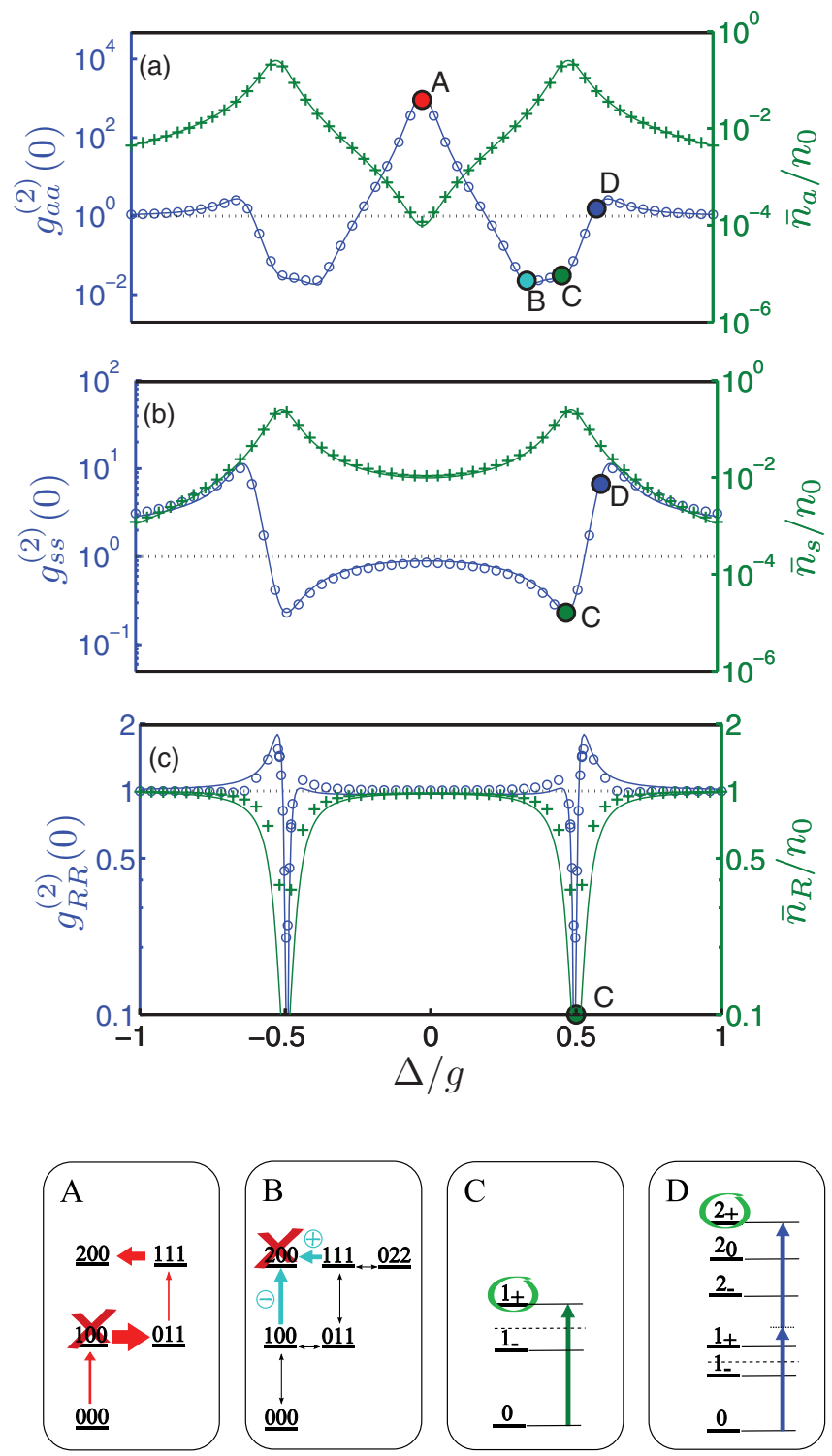

FIG. 2. (Color online) (a) Normalized average photon number (green "+") and photon-photon correlation function (blue "o") for driven mode $c_{a}$ as a function of laser detuning at zero temperature. Solid lines are calculated from the analytic model [see Eqs. (18)-(23)] and points show full numerical calculation. The average photon number is normalized by $n_{0}=(\Omega / \kappa)^{2}$. (b) Same as (a) for the undriven mode $c_{s}$, and (c) for the reflected field $c_{R}$. In all plots, we took $g / \kappa=20$ and $\gamma / \kappa=0.2$. Dots mark features seen at values of detuning (A) $\Delta / g=0$, (B) $\frac{1}{\sqrt{8}}$, (C) $\frac{1}{2}$, and (D) $\frac{\sqrt{6}}{4}$. The small discrepancy between the analytic and numerical results in (c) is due to the approximation $g / \kappa \gg 1$ to simplify the expressions in Eqs. (18)-(23). Bottom panels A-D illustrate the origin of these features as explained in the text. Suppression (enhancement) of the steady-state population of a specific level is indicated by a red $\mathrm{X}$ (green circle).

To gain insight into the two-photon correlation functions shown in Fig. 2, we develop an approximate analytic model for the system by considering only the six levels shown in Fig. 1(b). Assuming that the system is initially prepared in $|000\rangle$, these are the only levels significantly populated by weakly driving the $c_{a}$ mode. We make the 
ansatz [48]

$$
\begin{aligned}
|\psi\rangle= & A_{000}|000\rangle+A_{100}|100\rangle+A_{011}|011\rangle \\
& +A_{200}|200\rangle+A_{111}|111\rangle+A_{022}|022\rangle,
\end{aligned}
$$

and describe the dynamics by evolving $|\psi\rangle$ under the action of the non-Hermitian Hamiltonian, $\tilde{H}=H-i\left[\kappa c_{a}^{\dagger} c_{a}+\right.$ $\left.\kappa c_{s}^{\dagger} c_{s}+\frac{\gamma}{2} b^{\dagger} b\right]$. This approach allows us to evaluate intensities up to the order of $\Omega^{2}$ and two-point correlation up to the order of $\Omega^{4}$, since the neglected quantum jumps lead to higher-order corrections. By neglecting the typically small mechanical decay rate $\gamma \ll \kappa$, the amplitudes in Eq. (11) then satisfy

$$
\begin{gathered}
\dot{A}_{000}=0, \\
\dot{A}_{100}=-i \frac{g}{2} A_{011}-i \Omega A_{000}-\tilde{\kappa} A_{100}, \\
\dot{A}_{011}=-i \frac{g}{2} A_{100}-\tilde{\kappa} A_{011}, \\
\dot{A}_{200}=-i \frac{g}{\sqrt{2}} A_{111}-i \sqrt{2} \Omega A_{100}-2 \tilde{\kappa} A_{200}, \\
\dot{A}_{111}=-i \frac{g}{\sqrt{2}} A_{200}-i g A_{022}-i \Omega A_{011}-2 \tilde{\kappa} A_{111}, \\
\dot{A}_{022}=-i g A_{111}-2 \tilde{\kappa} A_{022},
\end{gathered}
$$

where $\tilde{\kappa}=\kappa-i \Delta$. It is straightforward to solve Eqs. (12)(17) for the steady-state amplitudes (see Appendix B). To the lowest order in $\Omega / \kappa$, the mean occupation numbers are $\bar{n}_{a}=\left|\bar{A}_{100}\right|^{2}, \bar{n}_{s}=\left|\bar{A}_{011}\right|^{2}$, and $\bar{n}_{R}=\left|\bar{A}_{100}+i \Omega / \kappa\right|^{2}$, where $\bar{A}$ denote steady-state amplitudes. We obtain

$$
\begin{gathered}
\frac{\bar{n}_{a}}{n_{0}}=\frac{\kappa^{2}\left[R_{\kappa}(0)\right]^{1 / 2}}{R_{\kappa}\left(\frac{g}{2}\right)}, \\
\frac{\bar{n}_{s}}{n_{0}}=\frac{g^{2} \kappa^{2}}{4 R_{\kappa}\left(\frac{g}{2}\right)}, \\
\frac{\bar{n}_{R}}{n_{0}} \approx \frac{\left[R_{\kappa / 2}\left(\frac{g}{2}\right)\right]^{2}}{\left[R_{\kappa}\left(\frac{g}{2}\right)\right]^{2}},
\end{gathered}
$$

where $R_{K}(\omega)=\left[K^{2}+(\Delta-\omega)^{2}\right]\left[K^{2}+(\Delta+\omega)^{2}\right]$ and $n_{0}=$ $(\Omega / \kappa)^{2}$. From the factors $R_{K}(\omega)$ in the denominators (numerators) in these expressions, we obtain the positions of the resonances (antiresonances) in the average intracavity photon numbers, in excellent agreement with the numerical results shown in Fig. 2. Our six-level model also provides the equal-time correlations (see Appendix B),

$$
\begin{gathered}
g_{a a}^{(2)}(0)=\frac{R_{\kappa}\left(\frac{g}{\sqrt{8}}\right) R_{\kappa}\left(\frac{g}{2}\right)}{R_{\kappa}(0) R_{\kappa}\left(\frac{\sqrt{6}}{4} g\right)}, \\
g_{s s}^{(2)}(0)=\frac{2 R_{\kappa}\left(\frac{g}{2}\right)}{R_{\kappa}\left(\frac{\sqrt{6}}{4} g\right)}, \\
g_{R R}^{(2)}(0) \approx \frac{R_{\kappa}\left(\frac{g}{2}\right) R_{16 \kappa^{3} / g^{2}}\left(\frac{g}{2}-\frac{2 \kappa^{2}}{g}\right)}{\left[R_{\kappa / 2}\left(\frac{g}{2}\right)\right]^{2}} .
\end{gathered}
$$

Again, these expressions are in agreement with the features seen in the numerical results in Fig. 2. The positions of maxima and minima are seen directly by the arguments of the factors $R_{K}(\omega)$. Note that we assumed $g / \kappa \gg 1$ to obtain the simplified expressions in Eqs. (21)-(23), but we retained the shift of order $g(\kappa / g)^{2}$ in the argument in Eq. (23) because this shift is larger than the width of the antiresonance.

We now discuss each feature in Fig. 2 in terms of our six-level model together with the diagonal basis in Eqs. (4)-(7). First, at detuning $\Delta=0$ (point A in Fig. 2), we see $g_{a a}^{(2)}(0)>1$, indicating bunching. This is due to destructive interference that suppresses the population in $|100\rangle$ (panel A in Fig. 2), and can be understood as the system being driven into a dark state, $|d\rangle \propto g|000\rangle-\Omega|011\rangle$, similar to electromagnetically induced transparency (EIT) [17,54]. In the dark state, $|011\rangle$ remains populated, allowing transitions to $|111\rangle$, which in turn is strongly coupled to $|200\rangle$. The net result is a relative suppression of the probability to have one photon compared to two photons in the driven mode, leading to bunching at $\Delta=0$. Second, at detuning $\Delta=g / \sqrt{8}$ (point B), mode $c_{a}$ shows antibunching due to a suppressed two-photon probability. Again, this is due to destructive interference, or the presence of a dark state in which $|200\rangle$ remains unpopulated (panel B). Third, at detuning $\Delta=\frac{g}{2}$ (point $\mathrm{C}$ ), all modes show antibunching. This is due to a one-photon resonant transition $|0\rangle \rightarrow\left|1_{+}\right\rangle$(panel C). Finally, at detuning $\Delta=\frac{\sqrt{6}}{4} g$ (point D), both $c_{a}$ and $c_{s}$ show bunching due to a two-photon resonant transition $|0\rangle \rightarrow\left|2_{+}\right\rangle$(panel D).

\section{Absence of two-photon resonance at $\Delta=0$}

At first glance, the level diagram in Fig. 1 together with bunching in Fig. 2(a) suggest a two-photon resonance at zero detuning $\Delta=0$, where the energy of the eigenstate $\left|2_{0}\right\rangle$ is equal to the energy of two drive photons. However, as discussed above, the bunching at $\Delta=0$ arises entirely from the suppression of a one-photon population; further, we find that the expected two-photon resonance is canceled by interference. This can be seen from a second-order perturbative calculation of the two-photon Rabi frequency $\Omega_{0,2_{0}}^{(2)}$ for the transition $|0\rangle \rightarrow\left|2_{0}\right\rangle$. The two-photon state $\left|2_{0}\right\rangle$ can be populated by the drive $H_{\mathrm{dr}}=\Omega\left(c_{a}^{\dagger}+c_{a}\right)$ from state $|0\rangle$ via two intermediate one-photon eigenstates, $\left|1_{ \pm}\right\rangle$given by Eq. (5), with energies $\omega_{1_{ \pm}}=-\Delta \pm \frac{g}{2}$ in the rotating frame. The resulting Rabi frequency is

$$
\Omega_{0,2_{0}}^{(2)}=\sum_{n=1_{-}, 1_{+}} \frac{\left\langle 2_{0}\left|H_{\mathrm{dr}}\right| n\right\rangle\left\langle n\left|H_{\mathrm{dr}}\right| 0\right\rangle}{\omega_{n}},
$$

which vanishes at $\Delta=0$ as a consequence of destructive interference between the two amplitudes. The exact cancellation is lifted by including finite dissipation and the full spectrum; nonetheless, this simple argument shows that the expected two-photon resonance at $\Delta=0$ is strongly suppressed.

Further evidence of the absence of a two-photon resonance at $\Delta=0$ is the lack of bunching in the undriven mode in Fig. 2(b). If there were a two-photon resonance, one would expect that bunching should also occur in the undriven mode, since the state $\left|2_{0}\right\rangle$ involves both $c_{a}$ and $c_{s}$ modes. This is indeed the case at detuning $\Delta=\frac{\sqrt{6}}{4} g$ (see point D in Fig. 2), where both modes show bunching as a result of two-photon resonance. In contrast, we see no bunching in the undriven mode at $\Delta=0$. This supports our conclusion that the observed bunching at $\Delta=0$ arises from suppression of population in $|100\rangle$ due to interference, as discussed in Sec. III B, and 


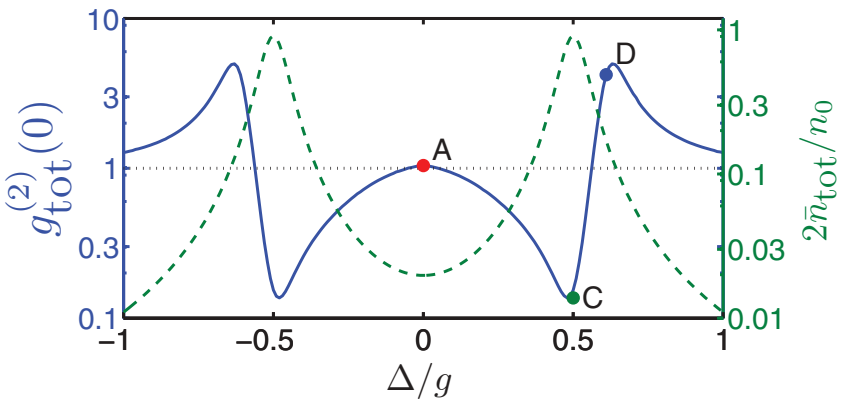

FIG. 3. (Color online) Average number (green dashed line) and intensity correlation (blue solid line) of total photon number in the coupled OM system. Parameters are the same as in Fig. 2, and dots mark the same detunings. One- and two-photon resonances are seen at $\mathrm{C}$ and $\mathrm{D}$, but we see no bunching in the total photon number at $\Delta=0$ (point A). This reflects the lack of two-photon resonance due to destructive interference [see Eq. (24)].

not from two-photon resonance. As discussed above, this interference does not suppress population in $|011\rangle$, so we do not expect bunching in the $c_{s}$ mode from this effect.

Finally, to confirm our intuitive picture, we plot the intensity correlation function, $g_{\text {tot }}^{(2)}(0)=\left\langle n_{\text {tot }}\left(n_{\text {tot }}-1\right)\right\rangle /\left\langle n_{\text {tot }}\right\rangle^{2}$, of the total photon number, $n_{\text {tot }}=n_{a}+n_{s}$, in the coupled $\mathrm{OM}$ system in Fig. 3. The probability to find one photon in the combined cavity is maximal at $\Delta / g= \pm \frac{1}{2}$ due to one-photon resonance. Similarly, we observe antibunching at point $\mathrm{C}$ and bunching at point $\mathrm{D}$ due to interference and two-photon resonance, respectively, as discussed in Sec. III B. However, we find neither bunching nor antibunching at $\Delta=0$, demonstrating the absence of a two-photon resonance despite the fact that $\left|2_{0}\right\rangle$ lies at twice the drive frequency.

\section{Finite temperature}

So far in our analysis we have focused on the case where the mechanical system is prepared in its vibrational ground state, $\left|0_{m}\right\rangle$. This condition can be achieved using high-frequency resonators operated at cryogenic temperatures [14], and in the limit of weak driving $\Omega / \kappa \ll 1$ such that optical heating of the mechanical mode can be neglected. The mechanical ground state could also be prepared using OM cooling [6-16], using an optical mode that is far detuned from the ones we consider here for nonlinear interactions [30]. Nonetheless, in the following, we extend our analytic treatment to the case of finite temperature, and show that many of the nonclassical features are robust even in the presence of small but finite thermal occupation of the mechanical mode.

To generalize our previous results, we now consider a finite equilibrium occupation number $N_{\text {th }}>0$ of the mechanical mode, but still assume that $\gamma\left(N_{\text {th }}+1\right) \ll \kappa, g$. Within this approximation, we proceed as above, and make a similar sixlevel ansatz as in Eq. (11) for each phonon number $n$,

$$
\begin{aligned}
\left|\psi_{n}\right\rangle= & A_{0,0, n}|0,0, n\rangle+A_{1,0, n}|1,0, n\rangle \\
& +A_{0,1, n+1}|0,1, n+1\rangle+A_{2,0, n}|2,0, n\rangle \\
& +A_{1,1, n+1}|1,1, n+1\rangle+A_{0,2, n+2}|0,2, n+2\rangle,
\end{aligned}
$$

where $\left|\psi_{n}\right\rangle$ includes states up to two photons that are connected by the weak drive and coupling $g$, starting from the state
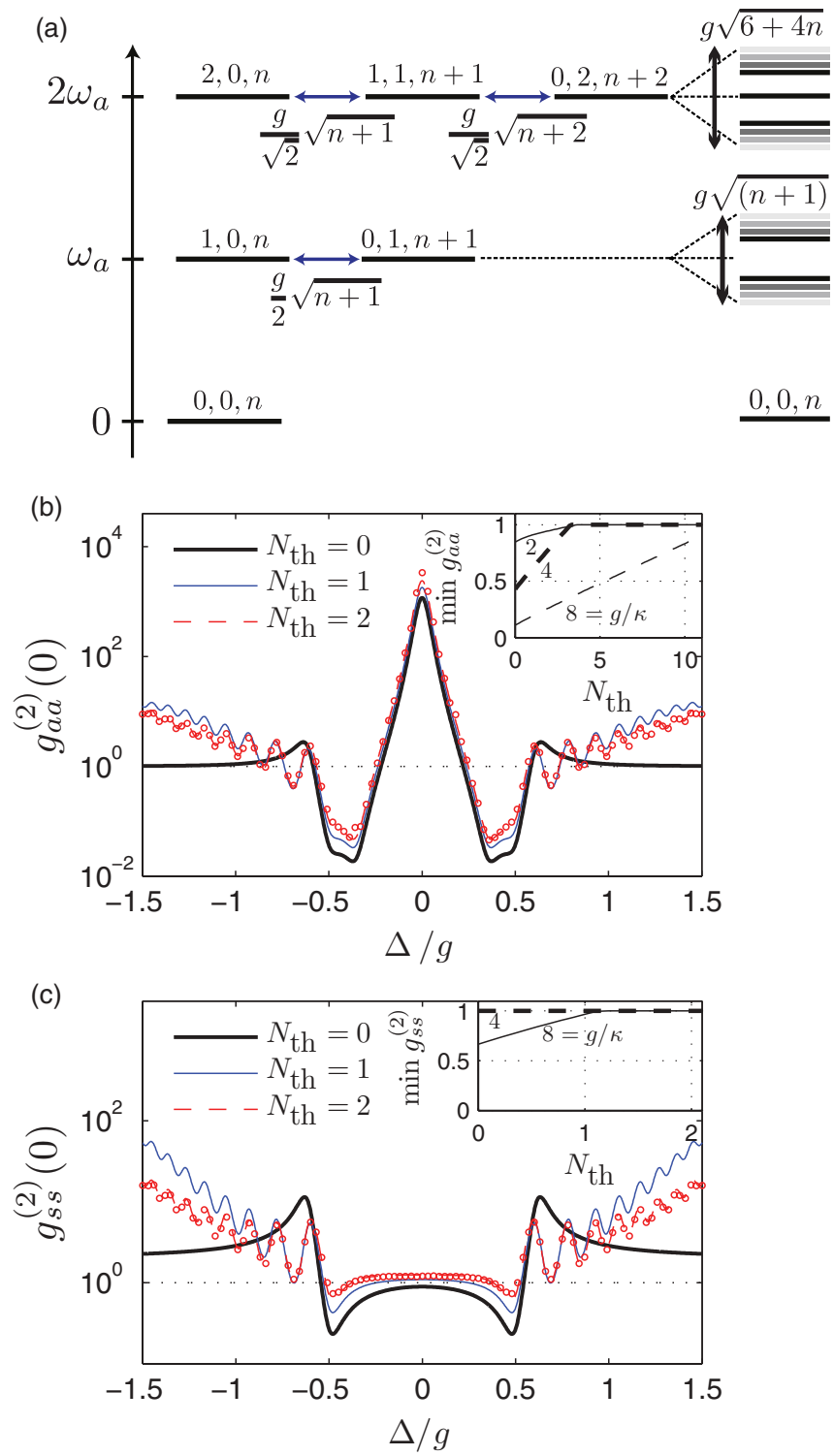

FIG. 4. (Color online) Correlation functions for finite temperature. (a) Level diagram showing the six states populated by the drive from level $|0,0, n\rangle$ (left), and the associated eigenmodes (right) with $n$-dependent splittings. (b) Driven mode correlation function $g_{a a}^{(2)}(0)$ for thermal mechanical occupation $N_{\text {th }}=0,1,2$. Solid lines show the analytic calculation with $\gamma \rightarrow 0$, and dots show the full numerical results for $N_{\text {th }}=2$ only. The inset shows the minimal $g_{a a}^{(2)}(0)$ as a function of $N_{\text {th }}$ for several coupling strengths. (c) Same as (b) for the undriven mode correlation function $g_{s s}^{(2)}(0)$. Parameters are $g / \kappa=20$ and (for numerics) $\gamma / \kappa=10^{-3}$.

$|00 n\rangle$. As shown in Fig. 4(a), the coupling between the states within each six-level subspace depends explicitly on the phonon number $n$. Following the same approach as above, the amplitudes in Eq. (25) evolve according to

$$
\begin{gathered}
\dot{A}_{0,0, n}=0, \\
\dot{A}_{1,0, n}=-i \frac{g}{2} \sqrt{n+1} A_{0,1, n+1}-i \Omega A_{, 00, n}-\tilde{\kappa} A_{1,0, n}, \\
\dot{A}_{0,1, n+1}=-i \frac{g}{2} \sqrt{n+1} A_{1,0, n}-\tilde{\kappa} A_{0,1, n+1},
\end{gathered}
$$




$$
\begin{gathered}
\dot{A}_{2,0,0}=-i g \sqrt{\frac{n+1}{2}} A_{1,1, n+1}-i \sqrt{2} \Omega A_{1,0, n}-2 \tilde{\kappa} A_{2,0, n}, \\
\dot{A}_{1,1, n+1}=-i g \sqrt{\frac{n+1}{2}} A_{2,0, n}-i g \sqrt{\frac{n+2}{2}} A_{0,2, n+2} \\
-i \Omega A_{0,1, n+1}-2 \tilde{\kappa} A_{1,1, n+1} \\
\dot{A}_{0,2, n+2}=-i g \sqrt{\frac{n+2}{2}} A_{1,1, n+1}-2 \tilde{\kappa} A_{0,2, n+2} .
\end{gathered}
$$

We solve for the steady-state amplitudes within each subspace $n$ and average the result over the initial thermal phonon distribution, assuming no coupling between subspaces due to the small phonon relaxation rate. We obtain the average photon numbers

$$
\bar{n}_{a}=\sum_{n} \zeta_{n}\left|\bar{A}_{1,0, n}\right|^{2}, \quad \bar{n}_{s}=\sum_{n} \zeta_{n}\left|\bar{A}_{0,1, n+1}\right|^{2}
$$

where $\zeta_{n}=e^{-\beta \hbar \omega_{m} n}\left(1-e^{-\beta \hbar \omega_{m}}\right)$ and $\beta^{-1}=k_{B} T$. Similarly, the $g_{i i}^{(2)}(0)$ functions are given by

$$
\begin{aligned}
& g_{a a}^{(2)}(0)=2 \sum_{n} \zeta_{n}\left|\bar{A}_{2,0, n}\right|^{2} / \bar{n}_{a}^{2}, \\
& g_{s s}^{(2)}(0)=2 \sum_{n} \zeta_{n}\left|\bar{A}_{0,2, n+2}\right|^{2} / \bar{n}_{s}^{2} .
\end{aligned}
$$

We provide the expressions for the steady-state amplitudes $\bar{A}_{2,0, n}$ and $\bar{A}_{0,2, n+2}$ in Appendix B.

In Fig. 4, we plot the correlation functions $g_{a a}^{(2)}(0)$ and $g_{s s}^{(2)}(0)$ for different thermal phonon numbers, $N_{\text {th }}$. Solid lines were calculated from the above analytic approach with $\gamma \rightarrow 0$, and we find excellent agreement with the full numerical results including small but finite $\gamma$ (dots, shown only for thermal occupation $N_{\text {th }}=2$ ). We see that the zero-temperature features such as antibunching survive at finite temperature for sufficiently strong coupling [30]. In the insets, we plot the minimum antibunching as a function of thermal occupation number for several ratios $g / \kappa$. Antibunching remains visible up to a critical thermal phonon number, set by $g / \kappa$, beyond which the contributions from different phonon numbers smear out the effect and antibunching vanishes. In addition, for detunings $|\Delta|>g / 2$, a series of new resonances appear in the correlation functions, and for small but finite occupation numbers we find new antibunching features that are absent for $N_{\text {th }}=0$. These new features can be understood from the $n$-dependent splitting of the one- and two-photon manifolds, as indicated in Fig. 4(a). For higher temperatures, the individual resonances start to overlap, and we observe an overall increase over a broad region of large positive and negative detunings due to the cumulative effect of different phonon numbers.

\section{DELAYED COINCIDENCE AND SINGLE-PHONON STATES}

In addition to the equal-time correlations discussed above, quantum signatures can also be manifested in photon intensity correlations with a finite time delay. We now turn to a discussion of delayed coincidence characterized by the two-time intensity correlations functions,

$$
g_{i i}^{(2)}(\tau)=\frac{\left\langle c_{i}^{\dagger}(0) c_{i}^{\dagger}(\tau) c_{i}(\tau) c_{i}(0)\right\rangle}{\left\langle c_{i}^{\dagger} c_{i}\right\rangle^{2}},
$$

for both driven and undriven modes, $i=a, s$. Expressing this correlation in terms of a classical light intensity $I, g^{(2)}(\tau)=$ $\langle I(\tau) I(0)\rangle /\langle I\rangle^{2}$, and using the Schwarz inequality, we obtain the inequalities $[48,49]$

$$
\begin{gathered}
g^{(2)}(\tau) \leqslant g^{(2)}(0), \\
\left|g^{(2)}(\tau)-1\right| \leqslant\left|g^{(2)}(0)-1\right| .
\end{gathered}
$$

Similar to the classical inequality $g^{(2)}(0)>1$ at zero delay, violation of either of these inequalities at finite delay is a signature of quantum light. We calculate the delayed coincidence correlation functions for both the driven and undriven modes.

\section{A. Driven mode}

The correlation function $g_{a a}^{(2)}(\tau)$ is shown in Fig. 5(a) for two values of the detuning $\Delta$. The most striking feature is the apparent vanishing of $g_{a a}^{(2)}(\tau)$ at several values of $\tau$ when the detuning is $\Delta=0$ [curve A in Fig. 5(a)]. These are due to Rabi oscillations at frequency $g / 2$ following the detection of a
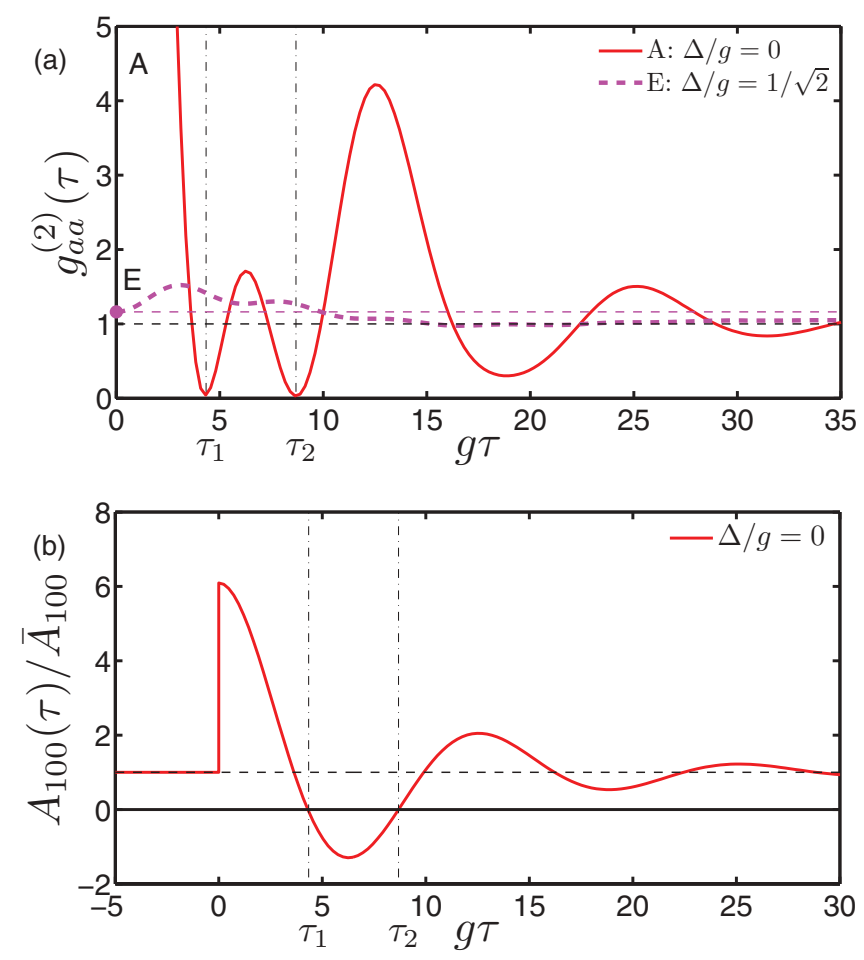

FIG. 5. (Color online) (a) Finite time delay intensity correlation function $g_{a a}^{(2)}(\tau)$ for detunings (A) $\Delta / g=0$ and (E) $\frac{1}{\sqrt{2}}$. Detuning for curve $\mathrm{A}$ is the same as marked in Fig. 2, while $\mathrm{E}$ shows a new effect not seen at equal times. Thin dashed line indicates the classical bound [see Eq. (36)] for curve E. (b) Evolution of amplitude $A_{100}$ (normalized by its steady-state value) at detuning $\mathrm{A}, \Delta / g=0$, after detecting a driven $c_{a}$ photon at $\tau=0$. Vertical dash-dotted lines mark delay times $\left(\tau_{1}, \tau_{2}\right)$ where this amplitude vanishes, resulting in the vanishing of $g_{a a}^{(2)}(\tau)$ in (a). Parameters are $g / \kappa=8$ and $\gamma / \kappa=0.02$. 
photon. This vanishing of the finite delay correlation function is reminiscent of wave-function collapse that occurs in a cavity containing an atomic ensemble [48], and while its origins are similar, there are important differences, as we now discuss.

We can understand the finite delay intensity correlations in terms of the simple six-level model discussed in the previous section. We extend this model to describe finite delay correlations by considering the effect of photodetection on the steady state of the system. Detection of a photon in the driven mode projects the system onto the conditional state [55],

$$
\left|\psi^{a}\right\rangle=\frac{c_{a}|\psi\rangle}{\| c_{a}|\psi\rangle \|}
$$

where $|\psi\rangle$ is given by Eq. (11) with steady-state amplitudes and $\|\cdot\|$ denotes normalization after the jump. The conditional state $\left|\psi^{a}\right\rangle$ has an increased amplitude $A_{100}$ after the jump [see jump at $\tau=0$ in Fig. 5(b)]. Following this initial photodetection, the amplitude $A_{100}$ subsequently undergoes Rabi oscillations with frequency $g / 2$, and decays back to its steady state at rate $2 \kappa$. For sufficiently large bunching at zero delay and strong coupling $g>\kappa$, the Rabi oscillations of the amplitude $A_{100}(\tau)$ can cause it to cross zero several times before it decays back to the steady state. As the probability to detect a second photon is dominated by $A_{100}$, its zeros are responsible for the zeros in the correlation function $g_{a a}^{(2)}(\tau)$ zero at these delay times.

The zeros in $g^{(2)}(\tau)$ appear similar to those exhibited in a cavity strongly coupled to an atomic ensemble $[48,49,56]$ or a single atom [57]. However, in stark contrast to the atomic case, the zeros in Fig. 5(a) are the result of Rabi oscillations following the initial quantum jump. This is qualitatively different from the atomic case, where the change in sign of the relevant amplitude (the analogy of $A_{100}$ ) occurs immediately after the jump itself, and the amplitude is damped back to the steady state at the atomic decay rate $\Gamma$, without Rabi oscillation. As a consequence, the vanishing correlation function in the atomic case occurs at a delay set by $\tau_{0} \sim \gamma^{-1} \ln C$, requiring only strong cooperativity $C=g^{2} / \kappa \gamma>1$ to be visible. On the other hand, the zeros in Fig. 5(a) occur at delay times set by $\tau \sim 1 / g$, requiring strictly strong coupling $g>\kappa$.

Before moving on to correlations of the undriven mode, we briefly discuss the correlations of the driven mode at the other value of detuning shown in Fig. 5(a). At detuning $\Delta=\frac{g}{\sqrt{2}}$ (curve E), which shows bunching at zero time, $g_{a a}^{(2)}(t)$ increases above its initial value at finite delay. This is a violation of the classical inequality in Eq. (36), and is an example of "delayed bunching," or an increased probability to detect a second photon at a finite delay time. A similar effect was recently studied in a single-mode OMS [43]. However, like the Rabi oscillations, the increased correlation function decays back to its steady-state value of 1 on the time scale of $\kappa^{-1}$.

\section{B. Heralded single-phonon states}

We now turn to a discussion of the delayed coincidence correlations of the undriven mode $c_{s}$. We note that correlations of the driven and undriven modes can be measured separately provided there is sufficient frequency resolution that is smaller than the mechanical frequency. The correlation function $g_{s s}^{(2)}(\tau)$

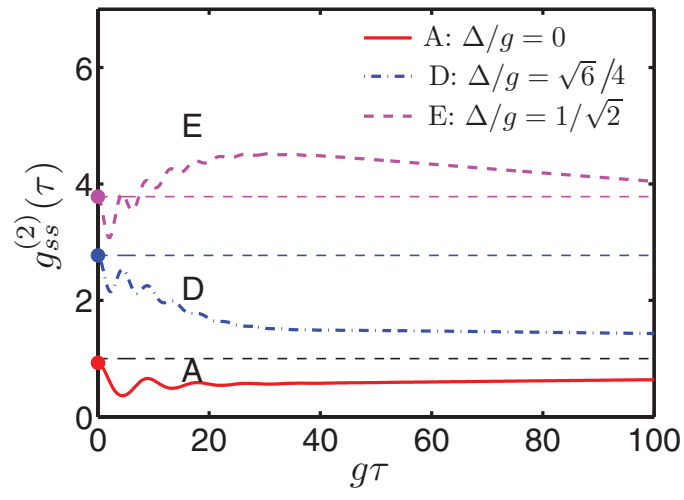

FIG. 6. (Color online) Finite time delay intensity correlation function $g_{s s}^{(2)}(\tau)$ for detunings (A) $\Delta / g=0$, (D) $\sqrt{6} / 4$, and (E) $\frac{1}{\sqrt{2}}$. Thin dashed lines indicate the classical bounds [see Eq. (36)]. Labels A, D, and E correspond to the same detunings marked in Figs. 2 and 5. Parameters are $g / \kappa=8$ and $\gamma / \kappa=0.02$.

of the undriven mode is shown in Fig. 6 for several values of detuning. Similar to the driven mode, the correlation function of the undriven mode exhibits Rabi oscillations that decay on the short optical time scale $1 / \kappa$. For detuning $\Delta=0$ and $\Delta / g=\frac{\sqrt{6}}{4}$ (curves A and D in Fig. 6), the correlation $g_{s s}^{(2)}(\tau)$ is described by our previous six-level model of Eqs. (12)-(17). However, at detuning $\Delta=\frac{g}{\sqrt{2}}$ (curve E), we see that $g_{s s}^{(2)}(\tau)$ has a long tail that decays on the much longer mechanical time scale $1 / \gamma$. This is due to the heralded preparation of a single phonon by detection of a photon in the undriven mode, as we now discuss.

The increase in delayed coincidence can be understood by extending the above analytic six-level model to account for the conditional state of the system after detection of a photon in the undriven mode. To do this, we simply add three additional states to the six-level ansatz in Eq. (11),

$$
|\psi\rangle=\cdots+A_{001}|001\rangle+A_{101}|101\rangle+A_{012}|012\rangle,
$$

since these are the states populated by detection of a $c_{s}$ photon from the original six states (see Fig. 7). Using the same approach as before, we obtain the following equations for

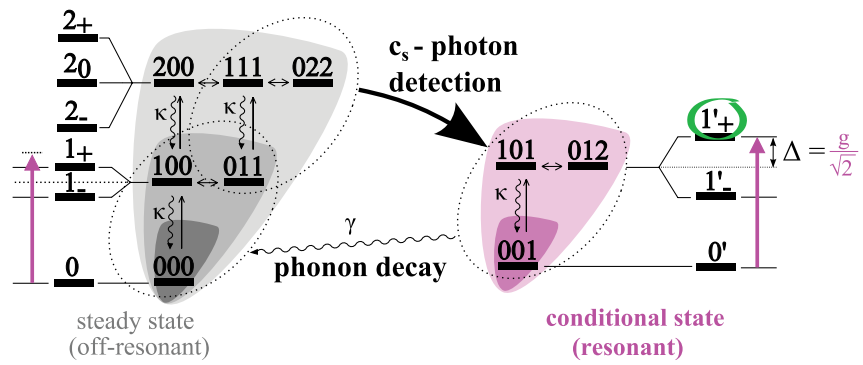

FIG. 7. (Color online) Effect of detection of a $c_{s}$ photon at detuning $\mathrm{E}\left(\Delta / g=\frac{1}{\sqrt{2}}\right)$. In the steady state (gray region on left), the drive is far off-resonant. However, after detection of a $c_{s}$ photon, the system jumps into the conditional subspace (pink region on right). Due to the presence of an extra phonon in this subspace, the drive is resonant and the probability to detect a second photon is much higher than in the steady state. This increased probability persists as long as the extra phonon, which decays slowly at rate $\gamma$. 
the amplitudes:

$$
\begin{gathered}
\dot{A}_{001} \approx-\frac{\gamma}{2} A_{001}, \\
\dot{A}_{101}=-i \frac{g}{\sqrt{2}} A_{012}-i \Omega A_{001}-\tilde{\kappa} A_{101}, \\
\dot{A}_{012}=-i \frac{g}{\sqrt{2}} A_{101}-\tilde{\kappa} A_{012},
\end{gathered}
$$

where we used $\gamma \ll \kappa$ and kept the leading term in Eq. (40). We obtain $g_{s s}^{(2)}(\tau)$ by solving these equations for initial conditions determined by the conditional state $\left|\psi^{s}\right\rangle$ after a quantum jump,

$$
\left|\psi^{s}\right\rangle=\frac{c_{s}|\psi\rangle}{\| c_{s}|\psi\rangle \|}
$$

which is a superposition of states $|001\rangle,|101\rangle,|012\rangle$ (see Appendix B for details), but in the limit of weak driving consist mainly of $|001\rangle$.

Detection of a photon in the undriven mode implies that the three-wave mixing interaction converted a photon from the driven mode into the undriven mode by simultaneously adding a phonon. The relevant three-level subspace after the jump (see Fig. 7) has a similar structure as in the steady state, but the presence of an extra phonon modifies the splitting of the one-photon states $\left|1_{ \pm}^{\prime}\right\rangle=(|101\rangle \pm|012\rangle) / \sqrt{2}$ to $\frac{g}{\sqrt{2}}$ (instead of $g / 2$ without a phonon). This changes the one-photon resonance condition for the drive to $\Delta=\frac{g}{\sqrt{2}}$. Therefore, at this value of the detuning, the process of exciting the system and emitting a single $c_{s}$ photon is off-resonant; while after the detection of a first $c_{s}$ photon, the system is prepared in $|001\rangle$, bringing it into resonance with the drive. This enhances the probability for subsequent excitation and emission of a second $c_{s}$ photon, increasing the correlation function at finite delay. The maximum delayed coincidence occurs after a delay of $\tau \sim 1 / \kappa$, when the photons have reached the metastable steady state in the conditional subspace with one extra phonon. Eventually, the delayed coincidence returns to its true steady-state value of one on the time scale $\tau \sim 1 / \gamma$, which is the mechanical decay time of the state $|001\rangle$. The long tails observed for other values of detuning (curves A and D) are also due to the presence of an extra phonon, but in these cases the system remains off-resonant after the initial $c_{s}$ photon and the effect is less pronounced. Note that the probability to detect a photon from the driven mode also increases in the conditional state, so a similar effect is seen in the delayed cross-correlation function $g_{a s}^{(2)}(\tau)$, where the photon in the undriven mode is detected first.

\section{REACHING STRONG COUPLING}

The nonclassical correlations predicted in this paper require strong optomechanical coupling, $g>\kappa$, as well as sideband resolution, $\omega_{m} \gg \kappa, \gamma$. While the combination of these conditions has not yet been demonstrated, several experimental efforts are currently directed at reaching this regime. By using micro- and nanofabricated OMSs such as microtoroids or photonic crystal beams, high-frequency mechanical systems with $\omega_{m} \approx 50 \mathrm{MHz}-5 \mathrm{GHz}$ can be combined with lowloss optical modes, such that the condition $\omega_{m} \gg \kappa \gg \gamma$ is satisfied $[11,23,35]$. At the same time, the mechanical system can already be prepared close to the quantum ground state by working at cryogenic temperatures. In microfabricated OMSs, single-photon couplings of about $g / \kappa \approx 0.001$ have been demonstrated [20,35,37]. The largest value to date of $g / \kappa \approx 0.007$ has been reached in photonic crystal-beam resonators [58], where colocalized optical and vibrational resonances are highly confined to maximize coupling, while the surrounding structure is engineered to minimize loss. Conversely, in cold-atomic experiments, the effective strong OM coupling regime has been reached [59], while sideband resolution remains a challenge [60].

There are several existing proposals for how to meet the challenge of $g / \kappa>1$ in the photonic crystal-beam setup. First, the single-photon optomechanical coupling can be increased by making use of nanoslots in the structure $[61,62]$ to further localize the electric field at the position of the mechanical mode. This could improve $g$ by a factor of 10 [46]. Second, numerical studies suggest that $\kappa$ can be further decreased by fine tuning the size and position of the slots in the photonic crystals $[63,64]$. Finally, new materials are currently being tested for an overall improvement of the OM properties of nanofabricated devices [65]. Thus by using these ultrahigh $Q$ photonic crystals or similar designs, an increase of $g / \kappa$ by a factor of $\sim 100$ is realistic. Note that once the strong-coupling condition has been achieved, the implementation of two- or multimode OMSs with adjustable tunneling, $2 J \sim \omega_{m}$, can be realized via evanescent field coupling, as has already been demonstrated in the weak-coupling regime $[23,35,50]$.

\section{CONCLUSIONS}

We have studied nonclassical intensity correlations in a driven, near-resonant optomechanical system with one mechanical and two optical modes. In the regime of strong coupling $g>\kappa$, this system allows for nonlinear quantum optics through a resonant three-mode interaction in which the exchange of two photons is mediated by a phonon. We have identified several different processes that can lead to nonclassical antibunching and delayed bunching, and we have derived a simple analytic model that allows us to describe and interpret photon-photon correlations in this system both at zero and at finite temperature. Our findings will be important as experiments approach the regime of strong OM coupling, and for potential applications of OMSs for quantum information processing. In particular, the long-lived correlation found for the undriven mode raises the intriguing possibility to exploit such a setup as a quantum memory. The generation of heralded single phonons on detection of a photon from the undriven mode may have implications for building OM quantum repeaters and quantum communication devices.

\section{ACKNOWLEDGMENTS}

We are grateful to Pierre Meystre, Norman Yao, and Nathalie de Leon for enlightening discussions. This work was supported by NSF, CUA, DARPA, NSERC, Harvard Purcell Fellowship, the Packard Foundation, the EU network AQUTE, and the Austrian Science Fund (FWF) through SFB FOQUS and the START Grant No. Y 591-N16. 


\section{APPENDIX A: DERIVATION OF REFLECTED MODE OPERATOR}

We obtain the reflected mode operator $c_{R}$ using input-output relations for a two-sided cavity. By assuming that the two end mirrors have the same transmittivity $(\propto \kappa)$, we can write the input-output relation for cavity mode $c_{a}$ on the driven mirror as

$$
c_{a, \mathrm{out}}=\sqrt{\kappa} c_{a}-c_{a, \mathrm{in}},
$$

where $c_{a, \text { in }}=-i \frac{\Omega}{\sqrt{\kappa}}$ is the incoming field operator on the mirror and $c_{a \text {,out }}$ is the output field operator. For direct comparison with $c_{a}$, we divide by $\sqrt{\kappa}$ and define the reflection mode operator as $c_{R} \equiv c_{a, \text { out }} / \sqrt{\kappa}=c_{a}+i \frac{\Omega}{\kappa}$.

\section{APPENDIX B: ANALYTIC MODEL}

In this Appendix, we provide the analytic solutions used to calculate one- and two-time correlation functions in the steady state. First, one-time correlations are calculated from the steady-state solutions of Eqs. (12)-(17). We set the time derivatives to zero and solve the equations iteratively, order by order, in the weak drive. This procedure yields

$$
\begin{gathered}
\bar{A}_{000} \approx 1, \\
\bar{A}_{100}=-i \alpha \frac{1}{1+4 x^{2}}, \\
\bar{A}_{011}=-\alpha \frac{2 x}{1+4 x^{2}}, \\
\bar{A}_{200}=-\frac{\alpha^{2}}{\sqrt{2}} \frac{1+2 x^{2}}{\left(1+4 x^{2}\right)\left(1+6 x^{2}\right)}, \\
\bar{A}_{111}=i \alpha^{2} \frac{2 x}{\left(1+4 x^{2}\right)\left(1+6 x^{2}\right)}, \\
\bar{A}_{022}=\alpha^{2} \frac{4 x^{2}}{\left(1+4 x^{2}\right)\left(1+6 x^{2}\right)},
\end{gathered}
$$

where $\alpha=\Omega / \tilde{\kappa}\left(|\alpha|^{2} \ll 1\right), x=g /(4 \tilde{\kappa})$, and $\tilde{\kappa}=\kappa-i \Delta$. Using these amplitudes, we can express all equal-time averages. The mean photon numbers are

$$
\begin{gathered}
\bar{n}_{a}=\left|\bar{A}_{100}\right|^{2}, \\
\bar{n}_{s}=\left|\bar{A}_{011}\right|^{2}, \\
\bar{n}_{R}=\left|\bar{A}_{100}+i \frac{\Omega}{\kappa}\right|^{2},
\end{gathered}
$$

and the photon-photon correlation functions are

$$
\begin{gathered}
g_{a a}^{(2)}(0)=\frac{2\left|\bar{A}_{200}\right|^{2}}{\left|\bar{A}_{100}\right|^{4}}, \\
g_{s s}^{(2)}(0)=\frac{2\left|\bar{A}_{022}\right|^{2}}{\left|\bar{A}_{011}\right|^{4}}, \\
g_{R R}^{(2)}(0)=\frac{\left|-\left(\frac{\Omega}{\kappa}\right)^{2}+2 i \frac{\Omega}{\kappa} \bar{A}_{100}+\sqrt{2} \bar{A}_{200}\right|^{2}}{\left|i \frac{\Omega}{\kappa}+\bar{A}_{100}\right|^{4}} .
\end{gathered}
$$

To leading order in $\kappa / g$, these yield Eqs. (18)-(23).

At finite temperature, we calculate steady-state amplitudes within each phonon subspace $n$ similarly in the ansatz of Eq. (25). Using the notation $R_{\kappa}(\omega)$ introduced in Sec. III B, the steady-state amplitudes within the subspace with $n$ phonons in the optical ground state are

$$
\begin{gathered}
\left|\bar{A}_{10 n}\right|^{2}=\frac{\Omega^{2} \sqrt{R_{\kappa}(0)}}{R_{\kappa}\left(\frac{g}{2} \sqrt{n+1}\right)}, \\
\left|\bar{A}_{01 n+1}\right|^{2}=\frac{\Omega^{2} g^{2}(n+1)}{4 R_{\kappa}\left(\frac{g}{2} \sqrt{n+1}\right)}, \\
\left|\bar{A}_{20 n}\right|^{2}=\frac{\Omega^{4} R_{\kappa}(g / \sqrt{8})}{R_{\kappa}(g \sqrt{(2 n+1) / 8}) R_{\kappa}\left(\frac{g}{2} \sqrt{n+1}\right)}, \\
\left|\bar{A}_{02 n+2}\right|^{2}=\frac{\Omega^{4} g^{4}(n+1)(n+2)}{32 R_{\kappa}(g \sqrt{(2 n+1) / 8}) R_{\kappa}\left(\frac{g}{2} \sqrt{n+1}\right)} .
\end{gathered}
$$

Two-time correlation functions are calculated similarly, using the conditional state after a jump [see Eqs. (38) and (43)] as the initial condition. For example, the unnormalized state after detection of a photon in the $c_{a}$ mode is $c_{a}|\psi\rangle=\bar{A}_{100}|000\rangle+\sqrt{2} \bar{A}_{200}|100\rangle+\bar{A}_{111}|011\rangle$. We solve Eqs. (12)-(14) for the amplitudes with this state as the initial condition. The finite delay correlation of the driven mode is

$$
g_{a a}^{(2)}(\tau)=\frac{\left|A_{100}(\tau)\right|^{2}}{\left|\bar{A}_{100}\right|^{4}},
$$

in good agreement with the numerics. The correlation of the undriven mode $g_{s s}^{(2)}(\tau)$ is calculated similarly. The unnormalized state after detection in the $c_{s}$ mode is $c_{s}|\psi\rangle=$ $\bar{A}_{011}|001\rangle+\bar{A}_{111}|101\rangle+\sqrt{2} \bar{A}_{022}|012\rangle$. Using this as the initial condition, we solve Eqs. (40)-(42) for the amplitudes in the conditional state. In the limit of $\gamma \ll \kappa$, we obtain

$$
g_{s s}^{(2)}(\tau)=\frac{\left|A_{012}(\tau)\right|^{2}}{\left|\bar{A}_{011}\right|^{4}} .
$$

[1] T. J. Kippenberg and K. J. Vahala, Science 321, 1172 (2008).

[2] F. Marquardt and S. Girvin, Phys. 2, 40 (2009).

[3] M. Aspelmeyer and K. Schwab, New J. Phys. 10, 095001 (2008).

[4] C. Genes, A. Mari, D. Vitali, and P. Tombesi, Adv. At. Mol. Opt. Phys. 57, 33 (2009).

[5] I. Favero and K. Karrai, Nature Photon. 3, 201 (2009).

[6] C. H. Metzger and K. Karrai, Nature (London) 432, 1002 (2004).
[7] S. Gigan, H. R. Böhm, M. Paternostro, F. Blaser, G. Langer, J. B. Hertzberg, K. C. Schwab, D. Bäuerle, M. Aspelmeyer, and A. Zeilinger, Nature (London) 444, 67 (2006).

[8] O. Arcizet, P.-F. Cohadon, T. Briant, M. Pinard, and A. Heidmann, Nature (London) 444, 71 (2006).

[9] D. Kleckner and D. Bouwmeester, Nature (London) 444, 75 (2006). 
[10] T. Corbitt, C. Wipf, T. Bodiya, D. Ottaway, D. Sigg, N. Smith, S. Whitcomb, and N. Mavalvala, Phys. Rev. Lett. 99, 160801 (2007).

[11] A. Schliesser, R. Rivière, G. Anetsberger, O. Arcizet, and T. J. Kippenberg, Nature Phys. 4, 415 (2008).

[12] J. D. Thompson, B. M. Zwickl, A. M. Jayich, F. Marquardt, S. M. Girvin, and J. G. E. Harris, Nature (London) 452, 72 (2008).

[13] D. J. Wilson, C. A. Regal, S. B. Papp, and H. J. Kimble, Phys. Rev. Lett. 103, 207204 (2009).

[14] A. D. O'Connell, M. Hofheinz, M. Ansmann, R. C. Bialczak, M. Lenander, E. Lucero, M. Neeley, D. Sank, H. Wang, M. Weides, J. Wenner, J. M. Martinis, and A. N. Cleland, Nature (London) 464, 697 (2010).

[15] J. D. Teufel, D. Li, M. S. Allman, K. Cicak, A. J. Sirois, J. D. Whittaker, and R. W. Simmonds, Nature (London) 471, 204 (2011).

[16] J. Chan, T. P. M. Alegre, A. H. Safavi-Naeini, J. T. Hill, A. Krause, S. Gröblacher, M. Aspelmeyer, and O. Painter, Nature (London) 478, 89 (2011).

[17] S. Weis, R. Rivière, S. Deléglise, E. Gavartin, O. Arcizet, A. Schliesser, and T. J. Kippenberg, Science 330, 1520 (2010).

[18] A. H. Safavi-Naeini, T. P. Mayer Alegre, J. Chan, M. Eichenfield, M. Winger, Q. Lin, J. T. Hill, D. E. Chang, and O. Painter, Nature (London) 472, 69 (2011).

[19] V. Fiore, Y. Yang, M. C. Kuzyk, R. Barbour, L. Tian, and H. Wang, Phys. Rev. Lett. 107, 133601 (2011).

[20] E. Verhagen, S. Deléglise, S. Weis, A. Schliesser, and T. J. Kippenberg, Nature (London) 482, 63 (2012).

[21] J. T. Hill, A. H. Safavi-Naeini, J. Chan, and O. Painter, arXiv:1206.0704.

[22] K. Stannigel, P. Rabl, A. S. Sørensen, P. Zoller, and M. D. Lukin, Phys. Rev. Lett. 105, 220501 (2010).

[23] A. H. Safavi-Naeini and O. Painter, New J. Phys. 13, 013017 (2011).

[24] C. A. Regal and K. W. Lehnert, J. Phys.: Conf. Ser. 264, 012025 (2011).

[25] J. M. Taylor, A. S. Sørensen, C. M. Marcus, and E. S. Polzik, Phys. Rev. Lett. 107, 273601 (2011).

[26] J. Zhang, K. Peng, and S. L. Braunstein, Phys. Rev. A 68, 013808 (2003).

[27] U. Akram, N. Kiesel, M. Aspelmeyer, and G. J. Milburn, New J. Phys. 12, 083030 (2010).

[28] D. E. Chang, A. H. Safavi-Naeini, M. Hafezi, and O. Painter, New J. Phys. 13, 023003 (2011).

[29] P. Rabl, Phys. Rev. Lett. 107, 063601 (2011).

[30] K. Stannigel, P. Komar, S. J. M. Habraken, S. D. Bennett, M. D. Lukin, P. Zoller, and P. Rabl, Phys. Rev. Lett. 109, 013603 (2012).

[31] M. Schmidt, M. Ludwig, and F. Marquardt, New J. Phys. 14, 125005 (2012).

[32] R. J. Schoelkopf and S. M. Girvin, Nature (London) 451, 664 (2008).

[33] S. Gröblacher, K. Hammerer, M. R. Vanner, and M. Aspelmeyer, Nature (London) 460, 724 (2009).

[34] J. D. Teufel, T. Donner, D. Li, J. W. Harlow, M. S. Allman, K. Cicak, A. J. Sirois, J. D. Whittaker, K. W. Lehnert, and R. W. Simmonds, Nature (London) 475, 359 (2011).
[35] M. Eichenfield, J. Chan, R. M. Camacho, K. J. Vahala, and O. Painter, Nature (London) 462, 78 (2009).

[36] T. Carmon and K. J. Vahala, Phys. Rev. Lett. 98, 123901 (2007).

[37] L. Ding, C. Baker, P. Senellart, A. Lemaitre, S. Ducci, G. Leo, and I. Favero, Appl. Phys. Lett. 98, 113108 (2011).

[38] S. Gupta, K. L. Moore, K. W. Murch, and D. M. Stamper-Kurn, Phys. Rev. Lett. 99, 213601 (2007).

[39] F. Brennecke, S. Ritter, T. Donner, and T. Esslinger, Science 322, 235 (2008).

[40] W. Marshall, C. Simon, R. Penrose, and D. Bouwmeester, Phys. Rev. Lett. 91, 130401 (2003).

[41] M. Ludwig, B. Kubala, and F. Marquardt, New J. Phys. 10, 095013 (2008).

[42] A. Nunnenkamp, K. Børkje, and S. M. Girvin, Phys. Rev. Lett. 107, 063602 (2011).

[43] A. Kronwald, M. Ludwig, and F. Marquardt, arXiv:1202.3674.

[44] J.-Q. Liao and C. K. Law, arXiv:1206.3085.

[45] H. Miao, S. Danilishin, T. Corbitt, and Y. Chen, Phys. Rev. Lett. 103, 100402 (2009).

[46] M. Ludwig, A. H. Safavi-Naeini, O. Painter, and F. Marquardt, Phys. Rev. Lett. 109, 063601 (2012).

[47] S. Basiri-Esfahani, U. Akram, and G. J. Milburn, New J. Phys. 14, 085017 (2012).

[48] H. J. Carmichael, R. J. Brecha, and P. R. Rice, Opt. Commun. 82, 73 (1991).

[49] R. J. Brecha, P. R. Rice, and M. Xiao, Phys. Rev. A 59, 2392 (1999).

[50] I. S. Grudinin, H. Lee, O. Painter, and K. J. Vahala, Phys. Rev. Lett. 104, 083901 (2010).

[51] M. Zhang, G. Wiederhecker, S. Manipatruni, A. Barnard, P. L. McEuen, and M. Lipson, Phys. Rev. Lett. 109, 233906 (2012).

[52] J. M. Dobrindt and T. J. Kippenberg, Phys. Rev. Lett. 104, 033901 (2010).

[53] H. K. Cheung and C. K. Law, Phys. Rev. A 84, 023812 (2011).

[54] M. D. Lukin, Rev. Mod. Phys. 75, 457 (2003).

[55] C. Gardiner and P. Zoller, Quantum Noise (Springer, Berlin, 2004).

[56] P. R. Rice and H. J. Carmichael, IEEE J. Quantum Electron. 24, 1351 (1988)

[57] D. E. Chang, A. S. Sørensen, E. A. Demler, and M. D. Lukin, Nature Phys. 3, 807 (2007).

[58] J. Chan, A. H. Safavi-Naeini, J. T. Hill, S. Meenehan, and O. Painter, Appl. Phys. Lett. 101, 081115 (2012).

[59] T. P. Purdy, D. W. C. Brooks, T. Botter, N. Brahms, Z.-Y. Ma, and D. M. Stamper-Kurn, Phys. Rev. Lett. 105, 133602 (2010).

[60] D. M. Stamper-Kurn, arXiv:1204.4351v1.

[61] J. T. Robinson, C. Manolatou, L. Chen, and M. Lipson, Phys. Rev. Lett. 95, 143901 (2005).

[62] M. Davanco, J. Chan, A. H. Safavi-Naeini, O. Painter, and K. Srinivasan, arXiv:1207.5020.

[63] M. Notomi, E. Kuramochi, and H. Taniyama, Opt. Express 16, 11095 (2008).

[64] Y. Tanaka, T. Asano, and S. Noda, J. Lightwave Technol. 26, 1532 (2008).

[65] C. Xiong, W. H. P. Pernice, X. Sun, C. Schuck, K. Y. Fong, and H. X. Tang, New J. Phys. 14, 095014 (2012). 